\title{
An Information-Theoretic Approach to PMU Placement in Electric Power Systems
}

\author{
Qiao Li, Student Member, IEEE, Tao Cui, Student Member, IEEE, Yang Weng, Student Member, IEEE, \\ Rohit Negi, Member, IEEE, Franz Franchetti, Member, IEEE, and Marija D. Ilić, Fellow, IEEE
}

\begin{abstract}
This paper presents an information-theoretic approach to address the phasor measurement unit (PMU) placement problem in electric power systems. Different from the conventional 'topological observability' based approaches, this paper advocates a much more refined, information-theoretic criterion, namely the mutual information (MI) between PMU measurements and power system states. The proposed MI criterion not only includes observability as a special case, but also rigorously models the uncertainty reduction on power system states from PMU measurements. Thus, it can generate highly informative PMU configurations. The MI criterion can also facilitate robust PMU placement by explicitly modeling probabilistic PMU outages. We propose a greedy PMU placement algorithm, and show that it achieves an approximation ratio of $(1-1 / e)$ for any PMU placement budget. We further show that the performance is the best that one can achieve, in the sense that it is NP-hard to achieve any approximation ratio beyond $(1-1 / e)$. Such performance guarantee makes the greedy algorithm very attractive in the practical scenario of multi-stage installations for utilities with limited budgets. Finally, simulation results demonstrate near-optimal performance of the proposed PMU placement algorithm.
\end{abstract}

Index Terms-Electric power systems, greedy algorithm, mutual information, phasor measurement unit, submodular functions.

\section{INTRODUCTION}

$\mathbf{S}$ YNCHRONIZED MEASUREMENT TECHNOLOGY (SMT) has been widely recognized as an enabler of the emerging real-time wide area monitoring, protection and control (WAMPAC) systems [1], [2]. Phasor measurement unit (PMU), being the most advanced and accurate instrument of SMT, plays a critical role in achieving key WAMPAC functionalities [3]. With better than one microsecond global positioning system (GPS) synchronization accuracy, the PMUs can provide highly synchronized, real-time, and direct measurements of voltage phasors at the installed buses, as well as current phasors of adjacent power branches. Such measurements are vital for the efficient and reliable operations of the power systems by improving the Situational Awareness (SA) of the grid operators, and facilitating synchronized and just-in-time (JIT) automated control actions [4], [5].

Manuscript received August 30, 2011; revised January 15, 2012, September 23, 2012; accepted October 16, 2012. Date of publication December 28, 2012; date of current version February 27, 2013.

The authors are with the Department of Electrical and Computer Engineering, Carnegie Mellon University, Pittsburgh, PA 15213 USA (email: liqiao02@gmail.com,; tcui@ece.cmu.edu; yweng@ece.cmu.edu; negi@ece.cmu.edu; franzf@ece.cmu.edu; milic@ece.cmu.edu).

Digital Object Identifier 10.1109/TSG.2012.2228242
Given the critical role of PMUs for the power system, it is important that these instruments are installed at carefully chosen buses, so as to maximize the 'information gain' on the system states. Currently, there is a significant performance gap between the existing research and the desired 'informative' PMU configuration [6], [7]. One particular reason is that most researches center around the topological observability criterion, which essentially specifies that power system states should be uniquely estimated using minimum number of PMU measurements [8]. Based on such criterion, many solutions were proposed, such as the ones based on mixed integer programming [9], [10], binary search [11], and metaheuristics [12], [13]. While it is true that all PMU configurations can monitor the power system states with similar accuracy once the system becomes fully observable, these PMU placement approaches can yield quite suboptimal results for the important and current situation, where the number of installed PMUs is far from sufficient to achieve full system observability. The reason is as follows. Firstly, the 'observability' criterion is very coarse, which specifies the information gain on system states as binary, i.e., either observable or non-observable. Such crude approximation essentially assumes that the states at different buses are completely independent (with exceptions for buses with zero injection), in that the knowledge of the state of a bus has zero information gain on the state of any other bus, as long as that bus is not 'observable'. This is clearly not the case for power systems, where the system states exhibit high correlations, due to the fundamental physical laws, such as KVL and KCL. Secondly, the observability approaches neglect important parameters of the power system, such as transmission line impedances, by focusing only on the binary connectivity graph. In this sense, if zero injection are not considered, the current researches is essentially the classic 'dominating set' problem [14], where a subset of buses in the system are selected, so that every bus is either in the subset, or a neighbor of the subset. Such over-simplification of the power system is very likely to result in suboptimal design and significant performance loss. For example, it has been shown in [6], [7] that PMU configurations can have large influence on the accuracy of state estimation, even though the observability result stays the same.

To overcome the performance limitation of current approaches, we advocate a much more refined, information-theoretic criterion to generate highly informative PMU placement configurations. Specifically, we rigorously model the 'information gain' achieved by the PMUs states as the Shannon mutual information (MI) [15] between the PMU measurements and the power system states. The MI criterion is very popular in 
statistics and machine learning literature [16], [17], which has been widely used in sensor placement problems. For power systems, we will show that maximizing the MI is equivalent to the minimizing the state estimation error covariance matrix, so that the MI formulation can directly contributes to improve state estimation results. Further, the MI metric includes the 'topological observability' in current research as a special case. Finally, the MI criterion can also model probabilistic PMU failures, to facilitate robust PMU placement configurations.

As a second contribution of this paper, we present a greedy PMU placement algorithm, and show that it can achieve (1$1 / e$ ) of the optimal information gain for any PMU budget $K$. We further prove that the approximation ratio is the best that one can achieve in practice, by showing that it is NP-hard to approximate with any factor larger than $(1-1 / e)$. Compared to existing approaches, the greedy algorithm not only achieves the best performance guarantee, but also can be easily extended to large-scale power systems. Further, the greedy PMU placement is very attractive in the practical scenario of multi-stage PMU installation, where utility companies prefer to install the PMUs over a horizon of multiple periods, due to limited budgets [18]. In such cases, utilities can simply adopt the greedy placement strategy, as the $(1-1 / e)$ approximation ratio holds for any $K$. On the other hand, existing multi-stage methods [18], [19] may incur significant performance loss if the multi-period budget changes unexpectedly.

Related work in power systems literature includes the entropy based approach [20], and the fuzzy clustering based approaches [21], [22]. In these approaches, the PMU buses were cleverly selected, so that either the 'information content' [20] of the contingency PMU response signals is maximized, or certain measure of 'dissimilarity' [21], [22] between PMU bus and non-PMU bus contingency response signals is minimized. Compared to these methods, the MI criterion and greedy placement method in this paper directly address the information-theoretic uncertainties in the power system states using the analytical DC model of the power system. Finally, the MI formulation and greedy method proposed in this paper are very general, which can be extended to many other complex real-world problems, such as sensor placement and feature selection [23], to generate good results with low computational complexity. Thus, we believe the approach proposed in this paper is of general interest to researchers in both power system and computational intelligence areas.

The remaining of this paper is organized as follows. Section II describes the power system and measurement models, and Section III formulates the optimal PMU placement problem. Section IV proposes the greedy PMU placement algorithm and analyzes its performance, and Section V demonstrates the numerical results. Finally, Section VI concludes this paper.

\section{System Model}

In this section, we formulate a Gaussian Markov random field (GMRF) model [24] for the system states, and describe the measurement models.

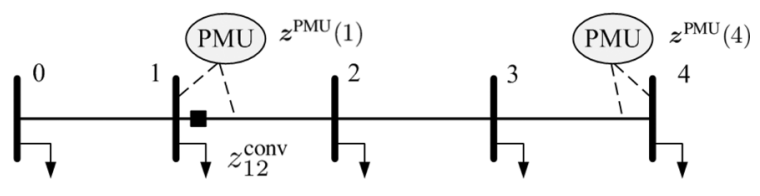

Fig. 1. The one line diagram of a power system with 5 buses. The square node represents the measurement of active power flow from bus 1 to 2 . Two PMUs are installed at bus 1 and 4 .

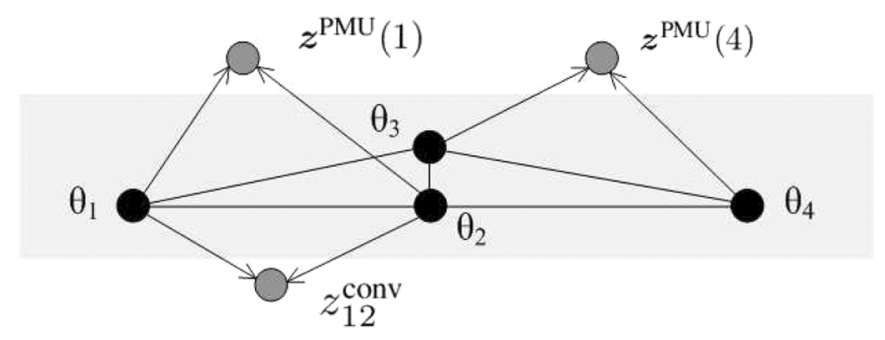

Fig. 2. The probabilistic graphical model for the non-reference bus angles in the power system in Fig. 1. The shaded region illustrates the GMRF for system states. The power injections are assumed to be independent.

\section{A. GMRF Model for Phasor Angles}

A DC power flow model [25] is assumed in this paper, where the power injection $P_{i}^{\text {inj }}$ at bus $i$ can be expressed as follows:

$$
P_{i}^{\mathrm{inj}}=B_{i i} \theta_{i}+\sum_{j \in \mathcal{N}_{i}} B_{i j} \theta_{j}
$$

In above, $\mathcal{N}_{i}$ is the set of neighboring buses of $i, B_{i j}$ is imaginary part of the nodal admittance matrix $Y$, and $\theta_{i}$ is the voltage phasor angle at bus $i$. The uncertainties in the power injection vector $\boldsymbol{P}^{\mathrm{inj}}$ can often be approximated as Gaussian by existing stochastic power flow methods [26]. In this paper, we assume that $\boldsymbol{P}^{\mathrm{inj}}$ is distributed as $\mathcal{N}\left(\boldsymbol{\mu}, \operatorname{Cov}\left(\boldsymbol{P}^{\mathrm{inj}}\right)\right)$. Denote bus 0 as the slack bus. We are only interested in the states at non-reference buses, as the angle of the slack bus can be uniquely specified by the non-reference bus angles, due to the law of power conservation. Write the non-reference bus angles in vector form as $\boldsymbol{\theta}=\left(\theta_{1}, \theta_{2}, \ldots, \theta_{N}\right)^{T}$. Note that the system states $\boldsymbol{\theta}$ are highly correlated statistically, due to the DC model in (1). Formally, the dependency of these variables are described by the following theorem:

Theorem 1: Assume the power system is fully connected. Under the DC model, $\boldsymbol{\theta}$ forms a GMRF with mean $B^{-1} \boldsymbol{\mu}$ and covariance matrix $\operatorname{Cov}(\boldsymbol{\theta})=B^{-1} \operatorname{Cov}\left(\boldsymbol{P}^{\mathrm{inj}}\right) B^{-1}$.

Proof: Since the power system is fully connected, the matrix $B$ is invertible [27]. Thus, the states can be calculated as $\boldsymbol{\theta}=B^{-1} \boldsymbol{P}^{\mathrm{inj}}$, from which the theorem follows.

Fig. 1 illustrates a 5-bus power system, with its GRMF model shown as the shaded region in Fig. 2. In this case, the GRMF is formed by connecting two-hop neighbors of the buses in the original power system, as the power injections are assumed to be independent. We next describe the PMU measurement model.

\section{B. Measurement Model}

1) Conventional Measurements: As the DC model is assumed in this paper, the conventional measurements only include the real power injection $\left\{z_{i}^{\text {conv }}\right\}$ and real power flow 
$\left\{z_{i j}^{\text {conv }}\right\}$. Under the DC model, these measurements can be described as follows:

$$
\begin{aligned}
& z_{i}^{\mathrm{conv}}=B_{i i} \theta_{i}+\sum_{j \in \mathcal{N}_{i}} B_{i j} \theta_{j}+e_{i}^{\mathrm{conv}} \\
& z_{i j}^{\mathrm{conv}}=-B_{i j}\left(\theta_{i}-\theta_{j}\right)+e_{i j}^{\mathrm{conv}}
\end{aligned}
$$

where $e_{i}^{\text {conv }}$ and $e_{i j}^{\text {conv }}$ are measurement noises, which are distributed as $\mathcal{N}\left(0, \kappa_{i}^{\text {conv }}\right)$ and $\mathcal{N}\left(0, \kappa_{i j}^{\text {conv }}\right)$, respectively.

2) PMU Measurements: A PMU placed at bus $i$ can measure both the voltage at bus $i$ and the currents of selected incident branches. This implies that the phasor angles of corresponding adjacent buses can also be directly calculated. Thus, we assume the following equivalent PMU measurement model. We associate a PMU placed at bus $i$ with a vector $z^{\mathrm{PMU}}(i)$, such that

$$
\begin{aligned}
& z_{i}^{\mathrm{PMU}}(i)=\theta_{i}+e_{i}^{\mathrm{PMU}} \\
& z_{i j}^{\mathrm{PMU}}(i)=\left(\theta_{i}-\theta_{j}\right)+e_{i j}^{\mathrm{PMU}}, \quad \forall j \in \mathcal{P}_{i}
\end{aligned}
$$

where $e_{i}^{\mathrm{PMU}}$ and $e_{i j}^{\mathrm{PMU}}$ are measurement noises with distribution $\mathcal{N}\left(0, \kappa_{i}^{\mathrm{PMU}}\right)$ and $\mathcal{N}\left(0, \kappa_{i j}^{\mathrm{PMU}}\right)$, respectively. $\mathcal{P}_{i} \subseteq \mathcal{N}_{i}$ is a subset of neighbors of bus $i$. This is because of the PMU channel limits, which imply that only a subset of adjacent branches can be monitored. The variances $\kappa_{i}^{\mathrm{PMU}}$ and $\kappa_{i j}^{\mathrm{PMU}}$ depend on various sources of uncertainties, such as the GPS synchronization, instrument transformers, A/D converters and cable parameters, which can be estimated appropriately [28]. Further, PMU failures can be modeled by assuming that each current measurement $z_{i j}^{\mathrm{PMU}}(i)$ outputs a failure message with probability $1-$ $a_{i j}^{\mathrm{PMU}}$, and similarly, each voltage measurement fails with probability $1-a_{i}^{\mathrm{PMU}}$, where $a_{i j}^{\mathrm{PMU}}$ and $a_{i}^{\mathrm{PMU}}$ are the availability of the current and voltage measurements, respectively.

As an illustration, Fig. 2 represents the probabilistic graph model for the measurement configuration in Fig. 1, where the gray nodes represents the measurement variables. From the figure, it is clear that the information gain of PMU measurements depends heavily on the placement buses. This will be formalized in the next section by the optimal PMU placement problem.

\section{OPTIMAL PMU PlaCEMENT: THEORETICAL FORMULATION}

The placement configuration of PMUs should be highly 'informative' to effectively monitor power system states. In this paper, we advocate an information-theoretic criterion to assess the 'information gain' that can be obtained from the PMU measurements. Specifically, we model the uncertainties in the system states as the Shannon entropy [15]:

$$
H(\boldsymbol{\theta})=\sum_{\boldsymbol{\theta}}-p(\boldsymbol{\theta}) \log p(\boldsymbol{\theta})
$$

where $p(\boldsymbol{\theta})$ is the probability mass function (pmf) of $\boldsymbol{\theta}$. In this paper, we assume that the entropies of all variables are calculated after quantization with a sufficiently small step size $\Delta$. This is motivated by finite accuracy of the meters in power systems. Notice that even though the $H(\boldsymbol{\theta})$ is defined from discrete distribution, it can be efficiently assessed numerically using the following well-known approximation:

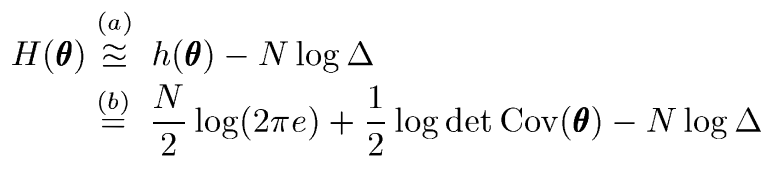

where $h(\boldsymbol{\theta})$ is the standard Shannon differential entropy [15] for the continuous version of random variable $\boldsymbol{\theta}$. $(a)$ is because of the approximation in [15], pp. 247-248, Theorem 8.3.1, and (b) is because of the GMRF model in Theorem 1 and the standard result of differential entropy for Gaussian variables in [15], pp. 249-150, Theorem 8.4.1. Denote $\mathcal{S}$ as the set of PMU configurations, where each element $s=\left\{i, \mathcal{P}_{i}\right\}$ in $\mathcal{S}$ corresponds to a candidate PMU configuration in (4) and (5). We remind the reader that $\mathcal{P}_{i}$ is the set of neighboring buses of bus $i$, such that $j \in \mathcal{P}_{i}$ implies that measurement (5) is taken for the branch $(i, j)$. Note that our model is very general, which can be used to model PMU channel limits. The information gain of the PMU configuration $\mathcal{S}$ can be assessed by the entropy reduction due to PMU measurements $z^{\mathrm{PMU}}(\mathcal{S})$ :

$$
I\left(\boldsymbol{\theta} ; \boldsymbol{z}^{\mathrm{PMU}}(\mathcal{S})\right)=H(\boldsymbol{\theta})-H\left(\boldsymbol{\theta} \mid \boldsymbol{z}^{\mathrm{PMU}}(\mathcal{S})\right)
$$

where $I\left(\boldsymbol{\theta} ; \boldsymbol{z}^{\mathrm{PMU}}(\mathcal{S})\right)$ is the Shannon mutual information (MI) between PMU measurements and power system states, and $H\left(\boldsymbol{\theta} \mid \boldsymbol{z}^{\mathrm{PMU}}(\mathcal{S})\right)$ is the conditional entropy. Finally, when conventional measurements are considered, the uncertainty reduction corresponds to the conditional MI:

$$
\begin{aligned}
I\left(\boldsymbol{\theta} ; \boldsymbol{z}^{\mathrm{PMU}}(\mathcal{S}) \mid \boldsymbol{z}^{\mathrm{conv}}\right)=H(\boldsymbol{\theta} \mid & \left.\boldsymbol{z}^{\mathrm{conv}}\right) \\
& -H\left(\boldsymbol{\theta} \mid \boldsymbol{z}^{\mathrm{PMU}}(\mathcal{S}), \boldsymbol{z}^{\mathrm{conv}}\right)
\end{aligned}
$$

A numerical example on the evaluation of the MI function is included later in Section V.B. Notice that the MI criterion is widely adopted in the machine learning literature to generate highly informative sensor placement configurations [16]. For the PMU placement problem in power systems research, the MI criterion is intimately related to the power system observability and state estimation accuracy, which we elaborate as follows:

1) Observability: The MI criterion can include the popular observability criterion as a special case. To see this, assume there is no PMU failure and zero PMU measurement noise. We claim that the maximum information gain is achieved if and only if the power system is completely observable from PMU measurements. This can be clearly observed from (9), where the MI function is maximized if and only if $H\left(\boldsymbol{\theta} \mid \boldsymbol{z}^{\mathrm{PMU}}(\mathcal{S})\right)=0$, in which case the system states are deterministic given the PMU measurements $z^{\mathrm{PMU}}(\mathcal{S})$.

2) State Estimation: The MI function is directly related to the power system state estimation error. In fact, the conditional entropy $H\left(\boldsymbol{\theta} \mid z^{\mathrm{PMU}}\right)$ can be expressed as follows:

$H\left(\boldsymbol{\theta} \mid \boldsymbol{z}^{\mathrm{PMU}}\right) \approx \log \operatorname{det} \operatorname{Cov}(\boldsymbol{\theta}-\hat{\boldsymbol{\theta}})+\frac{N}{2} \log (2 \pi e)-N \log \Delta$ 
where $\hat{\boldsymbol{\theta}}$ is the Minimum Mean Square Error (MMSE) estimation of $\boldsymbol{\theta}$ given the PMU measurements $z^{\mathrm{PMU}}$. Since the entropy $H(\boldsymbol{\theta})$ is fixed, the maximization of $\mathrm{MI}$ is equivalent to minimization of the state estimation error, which is represented by the quantity $\log \operatorname{det} \operatorname{Cov}(\boldsymbol{\theta}-\hat{\boldsymbol{\theta}})$ in the above equation. Intuitively, $\log \operatorname{det} \operatorname{Cov}(\boldsymbol{\theta}-\hat{\boldsymbol{\theta}})$ specifies how 'peaked' the distribution of power system state estimation error behaves. In the statistics literature, such criterion is referred to as the ' $\mathrm{D}$-optimality' [16].

We are now ready to formulate the optimal PMU placement problem. Assume that there are a total of $K$ PMUs to be installed in the power system. The goal is to choose a subset of PMU configurations $\mathcal{S}^{\star}$ from a set of candidate PMU configurations, such that

$$
\mathcal{S}^{\star} \in \arg \max _{|\mathcal{S}| \leq K} F_{i}(\mathcal{S}), \quad i=1,2 .
$$

The objective functions are illustrated as follows:

3) PMU Measurements Only: In this case, the objective function associated with a PMU placement set $\mathcal{S}$ is

$$
F_{1}(\mathcal{S})=\frac{1}{T} \sum_{t=1}^{T} I_{t}\left(\boldsymbol{\theta} ; z^{\mathrm{PMU}}(\mathcal{S})\right)
$$

where the dependence on time index $t$ is because the power system states $\boldsymbol{\theta}$ may have time-dependent distribution $\mathcal{N}\left(B^{-1} \boldsymbol{\mu}_{t}, B_{t}^{-1} \operatorname{Cov}\left(\boldsymbol{P}_{t}^{\mathrm{inj}}\right) B_{t}^{-1}\right)$. Thus, the objective function in (12) describes the 'time averaged information gain' about the power system state over a time period of interest.

4) With Conventional Measurements: When conventional measurements are considered, the objective function should be similarly formulated by the conditional MI function, as follows:

$$
F_{2}(\mathcal{S})=\frac{1}{T} \sum_{t=1}^{T} I_{t}\left(\boldsymbol{\theta} ; z^{\mathrm{PMU}}(\mathcal{S}) \mid z^{\mathrm{conv}}\right)
$$

Note that it is possible that the time scales can be different in both cases, as the conventional measurements can have much slower sampling rate (on the order of minutes) than PMU measurements. Having formulated the optimal PMU placement problem, we will discuss the solutions in the next section.

\section{GReedy PMU Placement Method}

It is highly desired that the PMUs are optimally placed in the power system. However, the optimal solution is very hard to obtain, as the optimal PMU placement problem is NP-complete [29]. In this section, we propose a greedy PMU placement algorithm, and show that it can achieve the optimal performance guarantee among the class of polynomial time algorithms.

\section{A. Hardness Result}

Before presenting the greedy algorithm, we first demonstrate the hardness result. We extend the hardness result in [29], by showing that the optimal PMU placement problem is not only NP-hard to solve, but also NP-hard to approximate beyond the approximation ratio of $(1-1 / e)$ :
Theorem 2: Unless $P=N P$, there is no polynomial time algorithm for the optimal PMU placement problem in (11) with better approximation ratio than $(1-1 / e)$.

Proof: See in Appendix A.

we next propose a greedy PMU placement algorithm, which can achieve the $(1-1 / e)$ approximation ratio.

\section{B. Greedy PMU Placement}

The greedy PMU placement algorithm is shown in Algorithm 1. Compared to the optimal placement, the greedy algorithm has low complexity, and is easy to implement in large-scale systems. In each step, the algorithm chooses the next candidate PMU configuration that can achieve the largest 'marginal information gain', where the objective function $F(\cdot)$ can be chosen as either $F_{1}$ or $F_{2}$, depending on whether conventional measurements are included.

\section{Algorithm 1 Greedy PMU Placement}

1: Initialize: $\mathcal{S} \leftarrow \emptyset$;

2: for $k=1$ to $K$ do

3: $\mathcal{S} \leftarrow \mathcal{S} \cup\left\{s^{\star}\right\}$, where $s^{\star}$ solves the following:

$$
s^{\star}=\arg \max _{s \notin \mathcal{S}} F(\mathcal{S} \cup\{s\})
$$

\section{4: end for \\ 5: return $\mathcal{S}$}

The next theorem shows that the greedy algorithm can achieve the largest approximation ratio of $(1-1 / e)$.

Theorem 3: The greedy PMU placement in (1) can achieve an approximation ratio of $(1-1 / e)$ for both objective functions $F_{1}(\cdot)$ and $F_{2}(\cdot)$.

Proof: The proof is obtained by identifying a key property, submodularity, of the PMU placement problem. Detailed proof is in Appendix B.

We have the following remarks:

1) Optimality: Based on Theorem 2 and 3, we claim that the greedy algorithm can achieve the best performance guarantee that is possible. Further, compared to methods such as mixed integer programming [9], [10], binary search [11], or metaheuristics [12], [13], the greedy algorithm is not only the best in performance guarantees, but also can be easily implemented in large-scale systems, due to the low computation complexity.

2) Multi-Stage Installation: The greedy algorithm is very attractive in the case of multi-stage installations, where the utilities plan to install the PMUs over a horizon of multiple phases, due to the limited (and possibly uncertain) budgets. In such scenarios, the greedy algorithm can always achieve an approximation ratio of $(1-1 / e)$ for any given $K$, whereas fixed multistage planning algorithms may suffer from substantial performance loss when the budget $K$ changes unexpectedly.

3) Other Practical Constraints: The greedy algorithm can be easily adapted for real power systems by incorporating other practical installation constraints, such as mandatory PMU buses and heterogeneous installation costs. For mandatory PMU buses, one can extend the MI functions in (9) and (10) 
to conditional MI functions, where certain PMUs have already been installed at pre-specified buses. For heterogeneous installation costs, one can still implement the greedy algorithm with an augmented budget limit (other than the cardinality limit $K$ ). The implementation details in such cases will be addressed in future research.

Having formulated the greedy PMU placement algorithm and proved its optimality results, we will test it against other methods in standard IEEE test systems in the next section.

\section{Numerical Results}

This section demonstrates the performance of the greedy PMU placement algorithm, and compares it with results in the literature.

\section{A. System Description}

In the simulation, real power injections are assumed to be normally distributed, and independent across different buses [26] For each bus, the standard deviation of the real power injection is assumed to be $10 \%$ of the mean value. The mean values are obtained by properly scaling the case profile description of the standard test system [30]. Thus, the MI functions at different time slots are only different by a multiplicative factor. Each PMU measurement is assumed to fail independently with probability 0.03 [19]. The standard error of each PMU measurements is assumed to be $0.02^{\circ}$, whereas the standard error of each conventional measurement is assumed to be $0.57^{\circ}$. For comparison purpose, we consider the 'topological observability' based PMU placement configurations in [31] to represent the typical performance of observability based PMU configurations. The results in [31] are obtained based on solving mixed integer programming. All simulation results are obtained with MATLAB on an Intel Xeon E5540 CPU with 8 GB RAM. The computation time for all simulations are shown in Table I.

\section{B. Numerical Calculation of Mutual Information}

We next briefly demonstrate the numerical calculation procedure of the MI function in (9). After that, the functions $F_{1}$ and $F_{2}$ in (12) and (13) can be straightforwardly evaluated. For simplicity of demonstration, the following procedure assumes no PMU failure. The calculation is as follows:

1) Calculate the covariance matrix $\operatorname{Cov}(\boldsymbol{\theta})$ of the phasor angles $\boldsymbol{\theta}$ according to Theorem 1.

2) Calculate the conditional covariance matrix $\operatorname{Cov}\left(\boldsymbol{\theta} \mid z^{\mathrm{PMU}}(\mathcal{S})\right.$ based on the measurement model in (4) and (5).

3) The MI function can now be calculated as follows:

$I\left(\boldsymbol{\theta} ; \boldsymbol{z}^{\mathrm{PMU}}(\mathcal{S})\right)=\log \operatorname{det} \operatorname{Cov}(\boldsymbol{\theta})$

$$
-\log \operatorname{det} \operatorname{Cov}\left(\boldsymbol{\theta} \mid \boldsymbol{z}^{\mathrm{PMU}}(\mathcal{S})\right) .
$$

Notice that $\operatorname{Cov}\left(\boldsymbol{\theta} \mid z^{\mathrm{PMU}}(\mathcal{S})\right)$ corresponds to the error covariance matrix of the MMSE estimator with PMU measurements $z^{\mathrm{PMU}}(\mathcal{S})$. Thus, maximizing the MI function is equivalent to minimizing the state estimation error. For conventional measurements, the conditional MI function in (10) can be calculated by replacing the covariance matrices $\operatorname{Cov}(\boldsymbol{\theta})$ and $\operatorname{Cov}\left(\boldsymbol{\theta} \mid z^{\mathrm{PMU}}(\mathcal{S})\right)$ with the conditional covariance matrices $\operatorname{Cov}\left(\boldsymbol{\theta} \mid z^{\text {conv }}\right)$ and $\operatorname{Cov}\left(\boldsymbol{\theta} \mid z^{\mathrm{PMU}}(\mathcal{S}), z^{\text {conv }}\right)$, respectively. Finally, when PMU measurement failures are considered, the MI function can be obtained by taking expectation over the set of successful PMU measurements. We next illustrate the above calculation procedure with an example.

Example: Consider the five-bus power system in Fig. 2. Assuming that the impedance of each branch is $j 1.0$ per unit, we can write matrix $B$ as follows:

$$
B=\left(\begin{array}{cccc}
2 & -1 & 0 & 0 \\
-1 & 2 & -1 & 0 \\
0 & -1 & 2 & -1 \\
0 & 0 & -1 & 1
\end{array}\right)
$$

Further, we assume that the active power injection vector is $\boldsymbol{P}^{\mathrm{inj}}=(1,1,1,1)^{T}$ per unit, and that the power injections are independent Gaussian variables with standard deviation of 0.1 per unit. According to Theorem 1, the covariance matrix of $\theta$ can be calculated as follows:

$$
\begin{aligned}
\operatorname{Cov}(\boldsymbol{\theta}) & =B^{-1} \operatorname{Cov}\left(\boldsymbol{P}^{\mathrm{inj}}\right) B^{-1} \\
& =\left(\begin{array}{llll}
0.04 & 0.07 & 0.09 & 0.10 \\
0.07 & 0.13 & 0.17 & 0.19 \\
0.09 & 0.17 & 0.23 & 0.26 \\
0.10 & 0.19 & 0.26 & 0.30
\end{array}\right)
\end{aligned}
$$

Now we assume there is no PMU failure and consider the candidate PMU configuration $\mathcal{S}=\{4,\{3\}\}$. That is, a PMU is placed at bus 4 , which can measure the state at bus 4 and branch $(4,3)$. According to the PMU measurement model in (4) and (5), the cross-covariance matrix $\operatorname{Cov}\left(\boldsymbol{\theta}, z^{\mathrm{PMU}}(\mathcal{S})\right)$ is

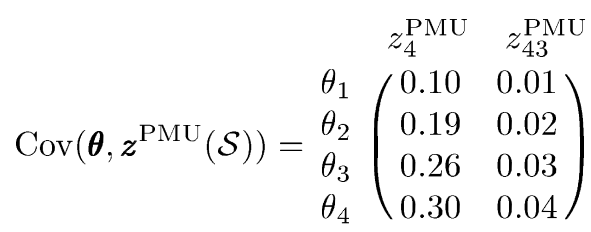

and the covariance matrix $\operatorname{Cov}\left(z^{\mathrm{PMU}}(\mathcal{S})\right)$ is

$$
\operatorname{Cov}\left(z^{\mathrm{PMU}}(\mathcal{S})\right)=\left(\begin{array}{cc}
0.30 & 0.04 \\
0.04 & 0.01
\end{array}\right)
$$

The conditional covariance matrix is calculated as in (20) and (21). Therefore, the MI function is

$$
\begin{aligned}
I\left(\boldsymbol{\theta} ; \boldsymbol{z}^{\mathrm{PMU}}(\mathcal{S})\right)= & \log \operatorname{det} \operatorname{Cov}(\boldsymbol{\theta}) \\
& -\log \operatorname{det} \operatorname{Cov}\left(\boldsymbol{\theta} \mid z^{\mathrm{PMU}}(\mathcal{S})\right) \\
= & 25.26
\end{aligned}
$$

\section{IEEE 14-Bus System}

1) PMU Measurements Only: For this case, the optimal PMU locations are calculated by an exhaustive search among all possible configurations to find the one that can maximize the MI objective functions. The PMU locations for both optimal and greedy placement for $K \leq 4$ are shown in Table II. From 
TABLE I

COMPUTATION TIME

\begin{tabular}{c|c}
\hline Test Systems & Time (Seconds) \\
\hline \hline IEEE 14-bus (PMU Only) & 0.298 \\
\hline IEEE 14-bus (Conventional) & 0.406 \\
\hline IEEE 30-bus (PMU Only) & 18.43 \\
\hline IEEE 57-bus (PMU Only) & 345.04 \\
\hline IEEE 57-bus (Conventional) & 13274 \\
\hline RTS-96 (PMU Only, 6 PMUs) & 0.1168 \\
\hline
\end{tabular}

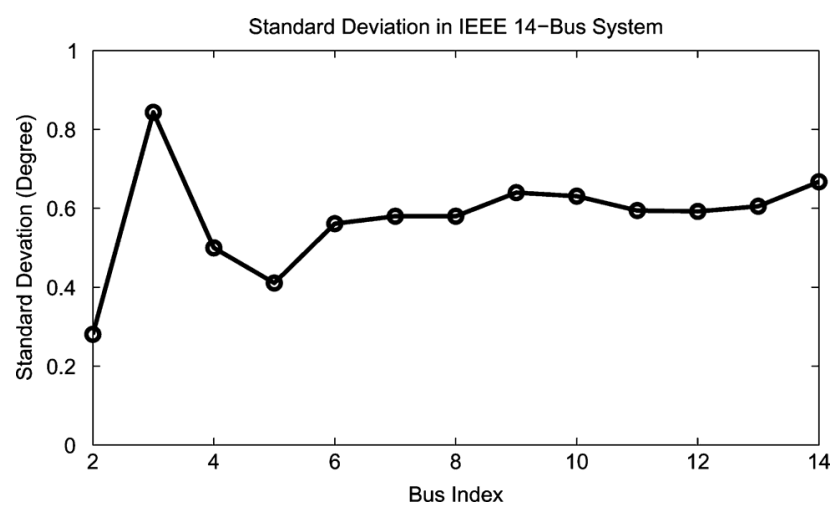

Fig. 3. Standard deviation of voltage angles in the IEEE 14-bus system.

the table, one can observe that the optimal PMU configuration can change significantly for different placement budget $K$. For example, when $K=2$, the optimal placement is $\{4,13\}$, whereas for $K=3$, the optimal placement is $\{4,6,9\}$. On the other hand, the greedy placement configuration $\mathcal{S}_{g}(K)$ always satisfy $\mathcal{S}_{g}(K-1) \subset \mathcal{S}_{g}(K)$. Thus, the greedy placement strategy is robust against the uncertainties in the placement budget $K$. To gain more insights on the placement results, we plot the standard deviations of the phasor angles at all non-reference buses in Fig. 3, which are obtained from the diagonal entries of the covariance matrix in Theorem 1. From the figure, one can observe that the state at bus 3 has the largest variance. However, bus 3 is not chosen as the first PMU bus, since, intuitively, it is connected to only two neighbors in the system (see the topology in [30]). Instead, bus 4 is chosen, since it has five neighbors.

Fig. 4(a) shows the normalized information gain for the IEEE 14-bus system with only PMU measurements. In the figure, the 'Upper Bound' curve is computed by the optimal PMU configuration assuming no PMU failure. Thus, it overestimates the information gain on the system states. One can easily observe the near optimal performance of the greedy PMU placement strategy, in that the 'Greedy' curve is very close to the 'Upper Bound'. Further, the greedy algorithm has a
TABLE II

PMU LOCATIONS FOR IEEE 14-BUS SYSTEM

\begin{tabular}{c|c|c|c|c}
\hline$K$ & $\begin{array}{c}\text { Optimal } \\
\text { (PMU Only) }\end{array}$ & $\begin{array}{c}\text { Greedy } \\
\text { (PMU Only) }\end{array}$ & $\begin{array}{c}\text { Optimal } \\
\text { (Conventional) }\end{array}$ & $\begin{array}{c}\text { Greedy } \\
\text { (Conventional) }\end{array}$ \\
\hline \hline 1 & 4 & 4 & 9 & 9 \\
\hline 2 & 4,13 & 4,13 & 4,13 & 9,6 \\
\hline 3 & $4,6,9$ & $4,13,9$ & $4,6,9$ & $9,6,4$ \\
\hline 4 & $4,6,9,13$ & $4,13,9,6$ & $4,6,9,13$ & $9,6,4,13$ \\
\hline
\end{tabular}

significant improvement on information gain compared to the conventional 'observability' based approach. For example, for $K=3$, the improvement is around $20 \%$ as compared to the observability based placement [31]. This clearly demonstrates the performance loss associated with the coarse observability based criterion. Finally, one can observe from the 'Upper Bound' curve that the maximum information gain has a 'diminishing marginal return' property, in that the marginal information gain tends to decrease as the number of installed PMUs in the power system grows. This also confirms the submodularity of the MI objective function.

2) With Conventional Measurements: In this case, real power flow measurements are obtained from the state estimation package of MATPOWER [32]. The detailed configuration is shown in Table III. The resulting PMU buses are shown in Table II. From the table, one can conclude that the optimal PMU placement is very vulnerable to the changes in the PMU placement budget $K$ than the PMU only case, as the configurations changes significantly as $K$ increases. On the other hand, the greedy algorithm is robust, as $\mathcal{S}_{g}(K-1) \subset \mathcal{S}_{g}(K)$ for any $K \geq 1$. The normalized information gain is shown in Fig. 4(b). We use the same configuration for the 'Observable' curve as the previous case. One can observe that the performance gain is larger compared to the case with only PMU measurements. This, again, confirms the conclusion that the pure topology based observability criterion cannot efficiently model the uncertainties in the power system states.

\section{IEEE 30-Bus System}

In order to further verify the near-optimal performance of the greedy algorithm, we next simulate the PMU placement in the IEEE 30-bus system. In this case, the size of the power system still allows us to compare against the optimal placement configuration. Due to space limitation, we only demonstrate the case with PMU measurements only. The results with conventional measurements are very similar. The PMU configuration is shown in Table IV, and the normalized information gain is shown in Fig. 5. From the figure, one can easily observe that the greedy algorithm achieves almost the same performance as the globally optimal configuration, and has significant gain over the

$$
\begin{aligned}
\operatorname{Cov}\left(\boldsymbol{\theta} \mid \boldsymbol{z}^{\mathrm{PMU}}(\mathcal{S})\right) & =\operatorname{Cov}(\boldsymbol{\theta})-\operatorname{Cov}\left(\boldsymbol{\theta}, \boldsymbol{z}^{\mathrm{PMU}}(\mathcal{S})\right) \operatorname{Cov}\left(z^{\mathrm{PMU}}(\mathcal{S})\right)^{-1} \operatorname{Cov}\left(z^{\mathrm{PMU}}(\mathcal{S}), \boldsymbol{\theta}\right) \\
& =\left(\begin{array}{cccc}
0.00429 & 0.00286 & 0.000000139 & 0.0000000522 \\
0.00286 & 0.00357 & 0.000000235 & 0.0000000957 \\
0.000000139 & 0.000000235 & 0.000000244 & 0.000000122 \\
0.0000000522 & 0.0000000957 & 0.000000122 & 0.000000122
\end{array}\right)
\end{aligned}
$$




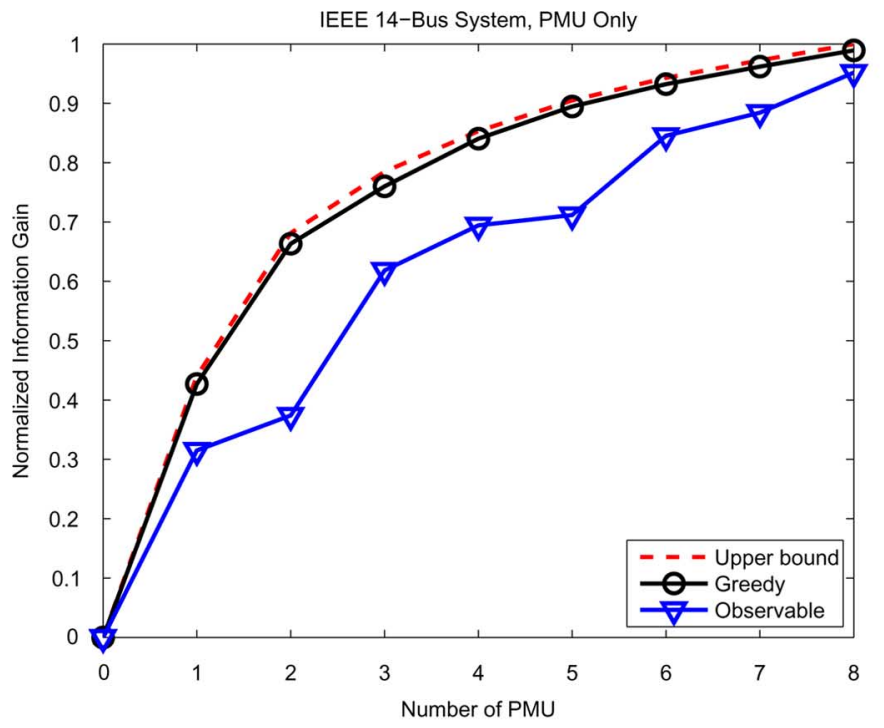

(a)

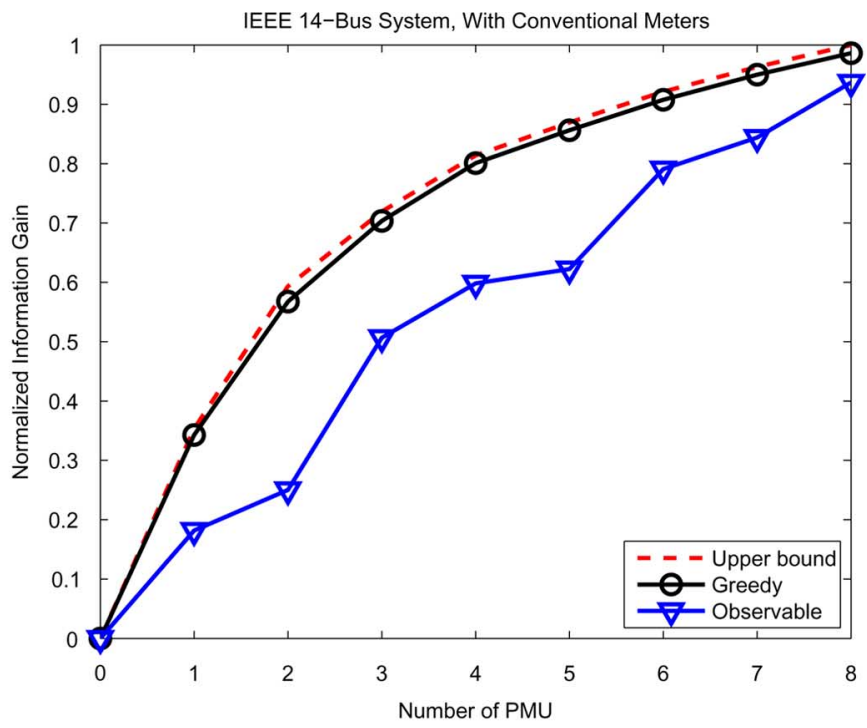

(b)

Fig. 4. Normalized information gain of different PMU placement schemes in the IEEE 14-bus system. (a) with PMU measurements only and (b) with conventional measurements.

TABLE III

CONFigurations OF CONVENTIONAL METERS

\begin{tabular}{c|l}
\hline Test Systems & Locations of Conventional Meters \\
\hline \hline IEEE 14-bus system & $1,2,3,6,8,1-2,4-2,5-2,2-3,5-4,5-6$, \\
& $11-6,4-7,4-9,7-9,9-10,6-13,12-13,9-4$ \\
\hline IEEE 57-bus system & $1,2,3,6,8,9,12,1-2,3-4,5-4,6-4,5-6$, \\
& $8-6,8-9,12-9,9-10,51-10,9-11,13-11,43-$ \\
& $11,10-12,13-12,17-12,13-14,1-15,14-15$, \\
& $1-16,1-17,4-18,18-19,19-20,20-21,22-$ \\
& $23,24-25,27-287-29,52-29,30-31,32-31$, \\
& $33-32,35-34,35-36,36-37,39-37,22-38$, \\
& $37-38,41-42,56-42,41-43,38-44,14-46$, \\
& $38-48,47-48,49-50,51-50,52-53,53-54$, \\
& $54-55,40-56,57-56,39-57$ \\
\hline
\end{tabular}

observability based PMU placement in [31]. This, again, verifies the near-optimal property of the greedy PMU placement method.

\section{E. IEEE 57-Bus System}

1) PMU Measurements Only: For the IEEE 57-bus system, it is computationally infeasible to obtain the optimal PMU configuration for large $K$. In such case, we only demonstrate the performance of the greedy PMU placement, and compare it against the observability based results in [31]. For the case with only PMU measurements, the resulting PMU configurations are shown in Table $\mathrm{V}$, and the normalized information gain is shown in Fig. 6(a). Similar to the IEEE 14-bus system, one can conclude that the greedy algorithm can achieve a significant information gain compared to the observability based criteria. This is because of the much more refined modeling of the MI function, which can effectively capture the remaining uncertainties in the states of the power system. Further, the information gain of the greedy placement curve also demonstrates the 'diminishing marginal return' property.

2) With Conventional Measurements: In this case, real power flow measurements are assumed to be configured at the branches

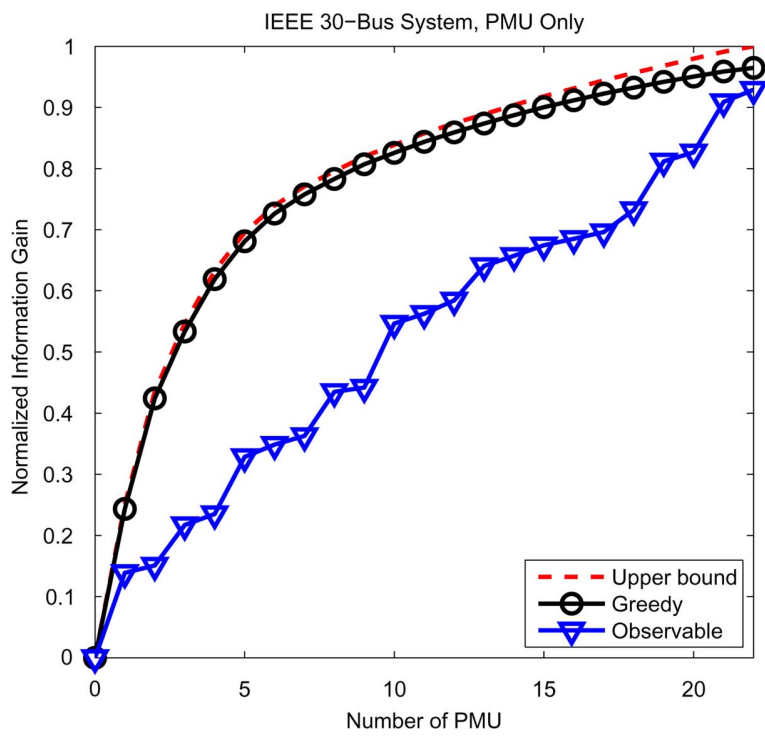

Fig. 5. Normalized information gain of different PMU placement schemes in the IEEE 30-bus system.

TABLE IV

PMU LOCATIONS FOR IEEE 30-Bus SySTEM

\begin{tabular}{c|c|c}
\hline$K$ & $\begin{array}{c}\text { Optimal } \\
\text { (PMU Only) }\end{array}$ & $\begin{array}{c}\text { Greedy } \\
\text { (PMU Only) }\end{array}$ \\
\hline \hline 1 & 27 & 27 \\
\hline 2 & 12,27 & 27,12 \\
\hline 3 & $6,12,27$ & $27,12,6$ \\
\hline 4 & $6,12,24,27$ & $27,12,6,24$ \\
\hline
\end{tabular}

and buses shown in Table III. The resulting greedy PMU configurations are shown in Table $\mathrm{V}$. The normalized information gain is shown in Fig. 6(b), where the greedy algorithm is compared against the same configuration in the previous case, Similar to 


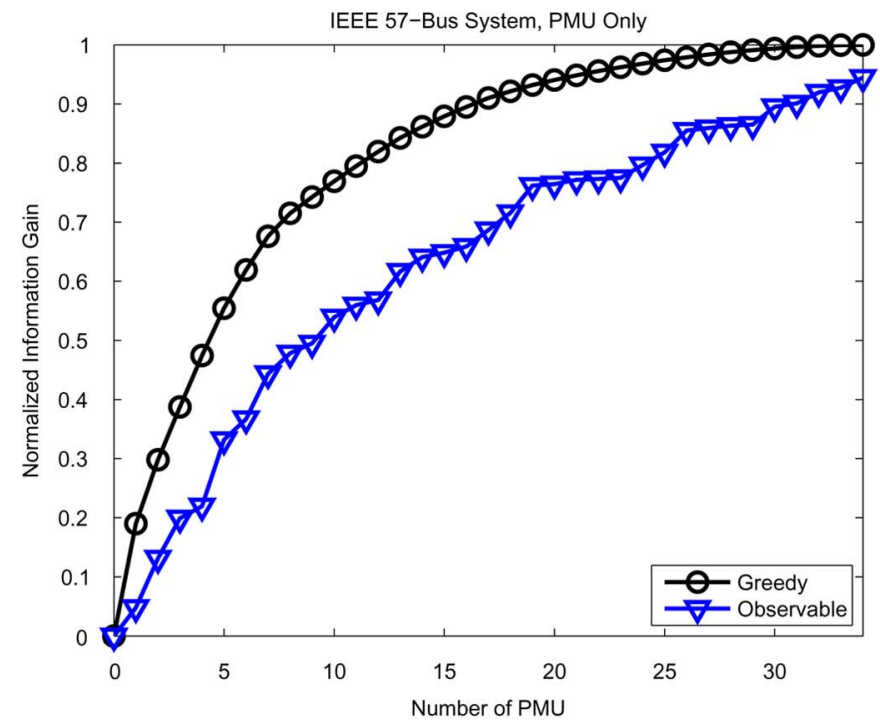

(a)

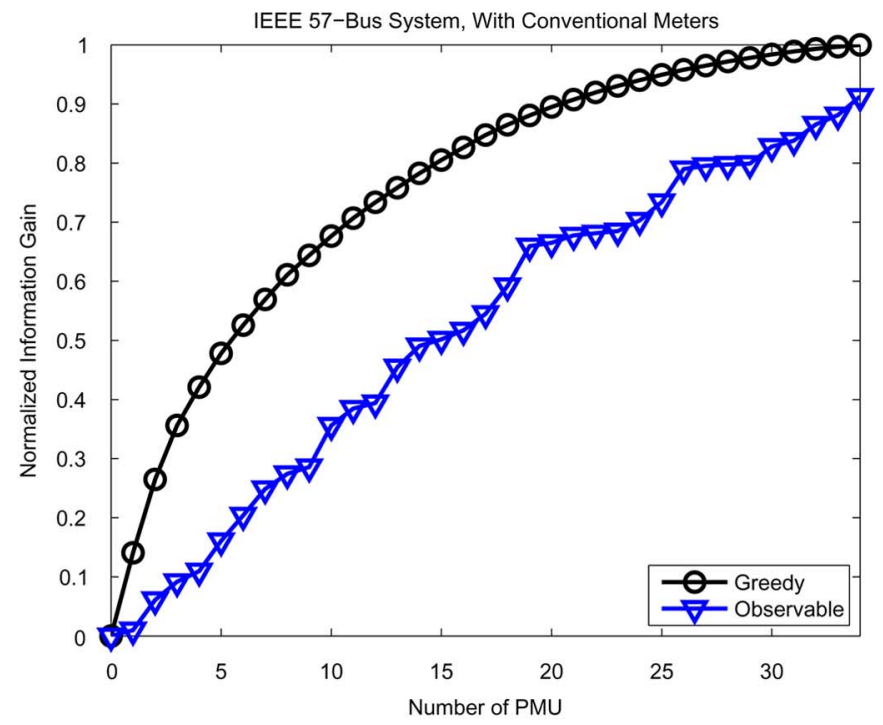

(b)

Fig. 6. Normalized information gain of different PMU placement schemes in the IEEE 57-bus system. (a) with PMU measurements only and (b) with conventional measurements.

TABLE V

PMU LOCATIONS FOR 57-BUS SYSTEM BY GREEDY ALGORITHM

\begin{tabular}{c|l}
\hline Test Scenarios & PMU Locations \\
\hline \hline IEEE 57-Bus (PMU Only) & $9,56,18,31,12,49,29,6$, \\
& $25,54,20,41,38,51,32$, \\
& $13,27,53,57,15,19,8,30$, \\
& $50,17,5,16,42,52,48,55$, \\
& $44,24,34$ \\
\hline IEEE 57-Bus (Conventional) & $56,31,19,12,54,49,25$, \\
& $41,32,9,29,18,50,6,20$, \\
& $57,27,53,38,30,13,42$, \\
& $51,17,55,52,5,34,24,43$, \\
& $16,44,8,10$ \\
\hline
\end{tabular}

the case without conventional measurements, one can observe the significant performance gain of the greedy algorithm.

\section{F. RTS-96 System}

We finally test the greedy algorithm in the RTS-96 system, and compare the placement configuration with the results in [21], which are obtained from fuzzy clustering based algorithms. Due to space limitation, we focus on the case with PMU measurements only. The placement configuration is shown in Table VI, where the PMUs are listed in the order of being selected by the greedy algorithm. Notice that in [21], the resulting PMU locations (cluster centers) are 109, 209, 303 for the case with three clusters, which are very similar to the first three greedy placement results. Both algorithms choose one bus in each area, which agrees with the symmetry in the test system topology. The normalized information gain is shown in 7. One can observe that the greedy algorithm achieves a slightly higher MI gain than the configuration in [21], due to the different PMU selection in area 3 (309 instead of 303).

We next compare the greedy result with the six cluster result in [21], which generates the following configuration: 310 , $215,109,115,210,315$. Compared with the greedy result with $K=6$ in Table VI, one can observe that the result in [21]
TABLE VI

PMU LoCATIONS FOR RTS-96 SYSTEM BY GREEDY ALGORITHM

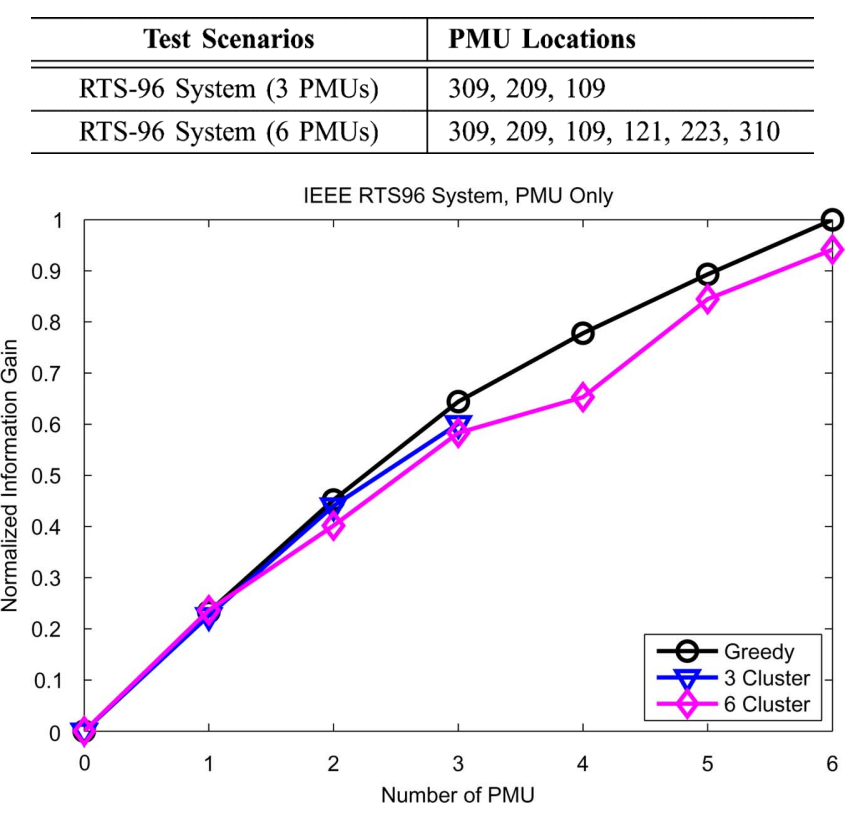

Fig. 7. Normalized information gain of different PMU placement schemes in the RTS-96 system.

changes significantly from the case with $K=3$. One reason is that the greedy selection for $K=6$ is based on $K=3$, whereas the fuzzy clustering algorithm in [21] computes $K=6$ independently from $K=3$. On the other hand, both algorithms place two PMUs in each area, which, again, agrees with the symmetry in the network topology. The information gain is shown in Fig. 7. Once gain, one can observe that the greedy algorithm achieves higher MI gain than the clustering based result. Thus, the greedy algorithm can achieve good performance with low computation complexity, and is robust against the PMU placement budget uncertainty. 


\section{CONCLUSION}

This paper proposed an information-theoretic approach to address the phasor measurement units (PMUs) placement for power system. Different from the topological observability based criterion in the literature, this paper proposed a much more refined, information-theoretic criterion, namely the mutual information (MI), as the PMU placement objective function. The proposed MI criterion can not only include the complete observability criterion as a special case, but also can accurately model the uncertainties in the system states. We further proposed a greedy PMU placement algorithm, and showed that it achieves an approximation ratio of $(1-1 / e)$ for any PMU budget $K$, which is the best guarantee among polynomial-time algorithms. Such performance guarantee makes the greedy algorithm attractive in the typical scenario of phased installations, as the performance is robust to the changes in the PMU budget. Finally, the performance of the proposed PMU placement algorithm was demonstrated by simulation results.

\section{APPENDIX A \\ PROOF OF THEOREM 2}

Proof: We prove the hardness result by constructing polynomial-time reduction of an arbitrary instance of the max $K$-cover problem [33] to a PMU placement problem. Thus, the hardness result easily follows from the $(1-1 / e)$ inapproximability of the max $K$-cover problem [33]. The $\max K$-cover problem is as follows. We are given a set of elements $\mathcal{U}=\{1,2, \ldots, N\}$, and a collection of sets $\mathcal{P}=\left\{\mathcal{P}_{1}, \mathcal{P}_{2}, \ldots, \mathcal{P}_{M}\right\}$, where each set $\mathcal{P}_{i}$ is a subset of $\mathcal{U}$. The task is to compute a subcollection of $K$ subsets $\mathcal{P}_{l_{1}}, \mathcal{P}_{l_{2}}, \ldots, \mathcal{P}_{l_{K}}$, such that the cardinality $\left|\cup_{k=1}^{K} \mathcal{P}_{l_{k}}\right|$ is maximized. Now, the reduction is as follows. Given a max $K$-cover problem instance, we construct a power system with $N+1$ buses, so that two buses $(i, j)$ are connected by a transmission line if and only if they both appear in a certain subset in $\mathcal{P}$. Further, we associate each subset $\mathcal{P}_{i}$ in $\mathcal{P}$ with a PMU, which is installed at a fixed (but can be arbitrarily chosen) bus in $\mathcal{P}_{i}$. Assume that the covariance matrix of the power injections $\boldsymbol{P}^{\mathrm{inj}}$ is $\operatorname{Cov}\left(\boldsymbol{P}^{\mathrm{inj}}\right)=\gamma B^{2}$, where $\gamma$ is a proper scalar that will be discussed later. Thus, according to Theorem 1, the resulting GMRF of $\boldsymbol{\theta}$ has covariance matrix $\gamma I$. Finally, choose $\gamma$ so that each discrete random variable $\theta_{i}$ has entropy 1 . Assume there is no PMU failure, and zero PMU measurement noise, we can write the MI objective function as follows:

$$
\begin{aligned}
I\left(\boldsymbol{\theta} ; \boldsymbol{z}^{\mathrm{PMU}}(\mathcal{S})\right) & =H(\boldsymbol{\theta})-H\left(\boldsymbol{\theta} \mid z^{\mathrm{PMU}}(\mathcal{S})\right) \\
& \stackrel{(a)}{=} N-H\left(\boldsymbol{\theta} \mid z^{\mathrm{PMU}}(\mathcal{S})\right) \\
& \stackrel{(b)}{=} N-\left|\overline{\cup_{k \in \mathcal{S}} \mathcal{P}_{k}}\right| \\
& =\left|\cup_{k \in \mathcal{S}} \mathcal{P}_{k}\right|
\end{aligned}
$$

where (a) is because the phasor angles are independent, and each has entropy 1, by construction. (b) is because given the PMU measurements, the uncertainties only remain at the phasor angles of the 'unobservable buses' $\overline{\cup_{k \in \mathcal{S}} \mathcal{P}_{k}}$. Denote $\mathcal{P}$ as the set of all possible PMU placement configurations. Thus, if we can solve the optimal PMU placement problem by maximizing $I\left(\boldsymbol{\theta} ; \boldsymbol{z}^{\mathrm{PMU}}(\mathcal{S})\right)$ subject to $|\mathcal{S}| \leq K$ beyond $(1-1 / e)$ in polynomial time, we can also achieve the same performance guarantee for the max $K$-cover problem in polynomial time, from which the theorem follows.

\section{APPENDIX B \\ PROOF OF THEOREM 3}

We now prove the $(1-1 / e)$ performance guarantee of Algorithm 1 . The key lies in identifying the submodular property of the PMU placement problem, which is a widely used concept in combinatorial optimizations.

\section{A. Introduction to Submodular Functions}

A set function $F$ is called submodular [34] if

$$
F(\mathcal{A} \cup\{k\})-F(\mathcal{A}) \geq F(\mathcal{B} \cup\{k\})-F(\mathcal{B})
$$

for any sets $\mathcal{A}$ and $\mathcal{B}$ such that $\mathcal{A} \subseteq \mathcal{B}$. Essentially, this is the 'diminishing marginal return' property, which, in the context of PMU placement, specifies that the marginal 'information gain' is decreasing as the number of installed PMU increases. A set function $F$ is nondecreasing if $\mathcal{S} \subseteq \mathcal{T}$ implies that $F(\mathcal{S}) \leq F(\mathcal{T})$, for all sets $\mathcal{S}$ and $\mathcal{T}$. The importance of submodularity can be seen by considering the following combinatorial optimization problem:

$$
\mathcal{S}^{\star}=\arg \max _{|\mathcal{S}| \leq K} F(\mathcal{S})
$$

For nondecreasing submodular functions, the following guarantee always holds for the greedy algorithm [34]:

Lemma 1: Let a set function $F(\cdot)$ be submodular, nondecreasing and $F(\emptyset)=0$. For any $K \geq 1$, denote $\mathcal{S}^{\star}(K)$ and $\mathcal{S}_{g}(K)$ as the optimal solution to the problem (28) and the solution obtained by Algorithm 1, respectively. Then,

$$
F\left(\mathcal{S}_{g}(K)\right) \geq(1-1 / e) F\left(\mathcal{S}^{\star}(K)\right)
$$

always holds. Thus, Algorithm 1 can achieve at least an approximation ratio of $(1-1 / e)$.

\section{B. Proof of Theorem 3}

Proof: We now prove the theorem by showing that the objective functions $F_{1}(\cdot)$ and $F_{2}(\cdot)$ for the optimal PMU placement problem satisfy all assumptions in Lemma 1 . We first fix time index $t$, and consider the case without conventional measurements. It is easy to see that $I_{t}\left(\boldsymbol{\theta} ; \boldsymbol{z}^{\mathrm{PMU}}(\emptyset)\right)=0$. We next verify (27) as follows:

$$
\begin{aligned}
& I\left(\boldsymbol{\theta} ; \boldsymbol{z}^{\mathrm{PMU}}(\mathcal{A} \cup s)\right)-I\left(\boldsymbol{\theta} ; \boldsymbol{z}^{\mathrm{PMU}}(\mathcal{A})\right) \\
\stackrel{(a)}{=} & I\left(\boldsymbol{\theta} ; \boldsymbol{z}^{\mathrm{PMU}}(s) \mid \boldsymbol{z}^{\mathrm{PMU}}(\mathcal{A})\right) \\
\stackrel{(b)}{=} & H\left(\boldsymbol{z}^{\mathrm{PMU}}(s) \mid \boldsymbol{z}^{\mathrm{PMU}}(\mathcal{A})\right) \\
& \quad-H\left(\boldsymbol{z}^{\mathrm{PMU}}(s) \mid \boldsymbol{\theta}, \boldsymbol{z}^{\mathrm{PMU}}(\mathcal{A})\right) \\
\stackrel{(c)}{=} & H\left(\boldsymbol{z}^{\mathrm{PMU}}(s) \mid \boldsymbol{z}^{\mathrm{PMU}}(\mathcal{A})\right)-H\left(\boldsymbol{z}^{\mathrm{PMU}}(s) \mid \boldsymbol{\theta}\right)
\end{aligned}
$$


In above, time index is omitted for notation simplicity. (a) follows from the chain rule of MI [15]. (b) follows from the definition of conditional MI. $(c)$ is because $z^{\mathrm{PMU}}(s)$ is conditionally independent of $z^{\mathrm{PMU}}(\mathcal{A})$ given $\boldsymbol{\theta}$, due to the measurement model in (4) and (5). This can also be clearly observed from the probabilistic graphical model in Fig. 1(b), where state variables $\boldsymbol{\theta}$ serve as the parent nodes of the PMU measurements in the 'Bayesian part' of the graph. Note that $H\left(z^{\mathrm{PMU}}(s) \mid z^{\mathrm{PMU}}(\mathcal{A})\right)$ in (33) is decreasing in $\mathcal{A}$, as conditioning always reduces entropy [15]. Since the second term in (33) is independent of $\mathcal{A},(27)$ follows easily. Finally, as conditional $\mathrm{MI}$ is always nonnegative, one can deduce from (31) that the MI function $I\left(\boldsymbol{\theta} ; \boldsymbol{z}^{\mathrm{PMU}}(\mathcal{A})\right)$ is also nondecreasing.

Now, the above analysis can be immediately extended to a time period of length $T$, where (27) holds for $F_{1}(\cdot)$ by summing up the inequalities corresponding to each time slot $t$, and then dividing both sides by $T$. Thus, we conclude that the claim holds for $F_{1}(\cdot)$. Similarly, an identical analysis can be carried out in the case with conventional measurements, where all functions in (31)-(33) hold when conditioned on conventional measurements.

\section{REFERENCES}

[1] V. Terzija, G. Valverde, D. Cai, P. Regulski, V. Madani, J. Fitch, S. Skok, M. Begovic, and A. Phadke, "Wide-area monitoring, protection, and control of future electric power networks," Proc. IEEE, vol. 99, no. 1, pp. 80-93, Jan. 2011.

[2] D. Bakken, A. Bose, C. Hauser, D. Whitehead, and G. Zweigle, "Smart generation and transmission with coherent, real-time data," Proc. IEEE, vol. 99, no. 6, pp. 928-951, Jun. 2011.

[3] A. G. Phadke and J. S. Thorp, Synchronized Phasor Measurements and Their Applications. New York: Springer, 2010.

[4] K. Sun, S. Likhate, V. Vittal, V. Kolluri, and S. Mandal, “An online dynamic security assessment scheme using phasor measurements and decision trees," IEEE Trans. Power Syst., vol. 22, no. 4, pp. 1935-1943, Nov. 2007.

[5] T. Overbye, P. Sauer, C. DeMarco, B. Lesieutre, and M. Venkatasubramanian, "Using PMU Data to Increase Situational Awareness," PSERC, Sep. 2010, Tech. Rep.

[6] M. Rice and G. Heydt, "Power systems state estimation accuracy enhancement through the use of PMU measurements," in Proc. IEEE PES Trans. Distr. Conf. Exhibition, May 2006, pp. 161-165.

[7] Q. Li, R. Negi, and M. Ilic, "Phasor measurement units placement for power system state estimation: A greedy approach," in Pow. IEEE Power Energy Society General Meeting, Jul. 2011, pp. 1-8.

[8] T. Baldwin, L. Mili, J. Boisen, M. B., and R. Adapa, "Power system observability with minimal phasor measurement placement," IEEE Trans. Power Syst., vol. 8, no. 2, pp. 707-715, May 1993.

[9] B. Xu and A. Abur, "Observability analysis and measurement placement for systems with PMUs," in Proc. IEEE Power Syst. Conf. Expo., Oct. 2004, vol. 2, pp. 943-946.

[10] B. Gou, "Optimal placement of PMUs by integer linear programming," Proc. IEEE Trans. Power Syst., vol. 23, no. 3, pp. 1525-1526, Aug. 2008.

[11] S. Chakrabarti and E. Kyriakides, "Optimal placement of phasor measurement units for power system observability," IEEE Trans. Power Syst., vol. 23, no. 3, pp. 1433-1440, Aug. 2008.

[12] B. Milosevic and M. Begovic, "Nondominated sorting genetic algorithm for optimal phasor measurement placement," Proc. IEEE Trans. Power Syst., vol. 18, no. 1, pp. 69-75, Feb. 2003.

[13] F. Aminifar, C. Lucas, A. Khodaei, and M. Fotuhi-Firuzabad, "Optimal placement of phasor measurement units using immunity genetic algorithm," IEEE Trans. Power Del., vol. 24, no. 3, pp. 1014-1020, Jul. 2009.

[14] V. Vazirani, Approximation Algorithms. New York: Springer, 2004.

[15] T. Cover and J. Thomas, Elements of Information Theory. : Wiley and Sons, 2006.

[16] D. J. C. MacKay, "Information-based objective functions for active data selection," Neural Comput., vol. 4, no. 4, pp. 590-604, 1992.
[17] A. Krause, A. Singh, and C. Guestrin, "Near-optimal sensor placements in gaussian processes: Theory, efficient algorithms and empirical studies," J. Mach. Learn. Res., vol. 9, pp. 235-284, June 2008.

[18] R. Nuqui and A. Phadke, "Phasor measurement unit placement techniques for complete and incomplete observability," IEEE Trans. Power Del., vol. 20, no. 4, pp. 2381-2388, Oct. 2005.

[19] F. Aminifar, M. Fotuhi-Firuzabad, M. Shahidehpour, and A. Khodaei, "Probabilistic multistage PMU placement in electric power systems," IEEE Trans. Power Del., vol. 26, no. 2, pp. 841-849, Apr. 2011.

[20] I. Kamwa and R. Grondin, "PMU configuration for system dynamic performance measurement in large, multiarea power systems," IEEE Trans. Power Syst., vol. 17, no. 2, pp. 385-394, May 2002.

[21] I. Kamwa, A. Pradhan, and G. Joos, "Automatic segmentation of large power systems into fuzzy coherent areas for dynamic vulnerability assessment," IEEE Trans. Power Syst., vol. 22, no. 4, pp. 1974-1985, Nov. 2007.

[22] I. Kamwa, A. Pradhan, G. Joos, and S. Samantaray, "Fuzzy partitioning of a real power system for dynamic vulnerability assessment," IEEE Trans. Power Syst., vol. 24, no. 3, pp. 1356-1365, Aug. 2009.

[23] A. Krause, "Optimizing Sensing: Theory and Applications," Ph.D. dissertation, Carnegie Mellon University, Pittsburgh, PA, Dec. 2008.

[24] M. He and J. Zhang, "A dependency graph approach for fault detection and localization towards secure smart grid," IEEE Trans. Smart Grid, vol. 2, no. 2, pp. 342-351, Jun. 2011.

[25] J. Grainger and W. Stevenson, Jr., Power System Analysis. New York: McGraw-Hill, 1994.

[26] A. Schellenberg, W. Rosehart, and J. Aguado, "Cumulant-based probabilistic optimal power flow (P-OPF) with Gaussian and Gamma distributions," IEEE Trans. Power Syst., vol. 20, no. 2, pp. 773-781, May 2005.

[27] G. Krumpholz, K. Clements, and P. Davis, "Power system observability: A practical algorithm using network topology," IEEE Trans. Power App. Syst., vol. PAS-99, no. 4, pp. 1534-1542, Jul. 1980.

[28] S. Chakrabarti, E. Kyriakides, and D. Eliades, "Placement of synchronized measurements for power system observability," IEEE Trans. Power Del., vol. 24, no. 1, pp. 12-19, Jan. 2009.

[29] D. Brueni and L. Heath, "The PMU placement problem," SIAM J. Discrete Math., vol. 19, no. 3, pp. 744-761, 2005.

[30] R. Christie, Power System Test Archive Aug. 1999 [Online]. Available: http://www.ee.washington.edu/research/pstca, [Online]. Available:

[31] B. Xu and A. Abur, "Optimal Placement of Phasor Measurement Units for State Estimation," PSERC, Oct. 2005, Tech. Rep.

[32] R. D. Zimmerman, C. E. Murillo-Snchez, and R. J. Thomas, "MATPOWER steady-state operations, planning and analysis tools for power systems research and education," IEEE Trans. Power Syst., vol. 26, no. 1, pp. 12-19, Feb. 2011.

[33] U. Feige, "A threshold of $\ln \mathrm{n}$ for approximating set cover," J. $A C M$, vol. 45, pp. 634-652, Jul. 1998.

[34] G. L. Nemhauser, L. A. Wolsey, and M. L. Fisher, "An analysis of approximations for maximizing submodular set functions," Math. Programm., vol. 14, pp. 265-294, 1978.

Qiao Li (S'07) received the B.Engg. degree from the Department of Electronics Information Engineering, Tsinghua University, Beijing China, in 2006. He received the M.S. degree from the Department of Electrical and Computer Engineering, Carnegie Mellon University, Pittsburgh, PA, in 2008. He is currently working toward the Ph.D. degree in the Department of Electrical and Computer Engineering, Carnegie Mellon University.

His research interests include monitoring and control in electrical energy systems, cyber-physical systems, and wireless networking.

Tao Cui (S'10) received the B.Eng. and M.Sc. degrees from Department of Electrical Engineering, Tsinghua University, Beijing China in 2006 and 2009, respectively. He is currently working toward the Ph.D. degree in the Department of Electrical and Computer Engineering, Carnegie Mellon University, Pittsburgh, PA.

His main research interests include power system computation, protection, analysis and control.

Yang Weng (S'08) received the B.Engg. degree in electrical engineering in 2006 from Huazhong University of Science and Technology, Wuhan, China, and the 
M.S. degree in statistics from University of Illinois at Chicago in 2009. Currently, he is a graduate student in the Department of Electrical and Computer Engineering at Carnegie Mellon University, Pittsburgh, PA.

His research interest includes power systems, smart grid, information theory, and wireless communications.

Rohit Negi (S'98-M'00) received the B.Tech. degree in electrical engineering from the Indian Institute of Technology, Bombay, in 1995. He received the M.S. and Ph.D. degrees from Stanford University, CA, in 1996 and 2000, respectively, both in electrical engineering.

Since 2000, he has been with the Electrical and Computer Engineering Department, Carnegie Mellon University, Pittsburgh, PA, where he is a Professor His research interests include signal processing, coding for communications systems, information theory, networking, cross-layer optimization, and sensor networks.

Dr. Negi received the President of India Gold Medal in 1995.

Franz Franchetti received the Dipl.-Ing. degree and the Ph.D. degree in technical mathematics from the Vienna University of Technology in 2000 and 2003, respectively.
He has been with the Vienna University of Technology since 1997. He is currently an Associate Research Professor with the Department of Electrical and Computer Engineering at Carnegie Mellon University. His research interests concentrate on the development of high performance DSP algorithms.

Marija D. Ilić (M'80-SM'86-F'99) is currently a Professor at Carnegie Mellon University, Pittsburgh, PA, with a joint appointment in the Electrical and Computer Engineering and Engineering and Public Policy Departments.

She is also the Honorary Chaired Professor for Control of Future Electricity Network Operations at Delft University of Technology in Delft, The Netherlands. She was an assistant professor at Cornell University, Ithaca, NY, and tenured Associate Professor at the University of Illinois at Urbana-Champaign. She was then a Senior Research Scientist in Department of Electrical Engineering and Computer Science, Massachusetts Institute of Technology, Cambridge, from 1987 to 2002. She has 30 years of experience in teaching and research in the area of electrical power system modeling and control. Her main interest is in the systems aspects of operations, planning, and economics of the electric power industry. She has co-authored several books in her field of interest.

Prof. Ilić is an IEEE Distinguished Lecturer. 